\title{
Marabou Stork Nightmares d'Irvine Welsh. Épuisement du récit réaliste et mise en récit de l'Histoire
}

\author{
Anne-Marie David
}

Université de Montréal

La littérature prend acte de la pauvreté ressentie du réel contemporain en optant pour l'épuisement, tandis que les discours déployés dans l'espace public, pour pallier cette même pauvreté, sont désormais le lieu d'une esthétisation et d'une narrativisation croissante. Or, si ces phénomènes sont complémentaires, il est difficile de les appréhender simultanément, puisqu'ils relèvent de domaines différents et prennent place dans des sphères parfois très éloignées. Le roman peut cependant, à certaines conditions, rendre compte 
de ce double jeu. Ainsi, Marabou Stork Nightmares, publié en 1995 par l'auteur écossais Irvine Welsh, fonctionne selon les dynamiques contradictoires qui viennent d'être énoncées. S'il y parvient, c'est au prix de son unité formelle : le récit est divisé en trames hétérogènes alternées, relevant de genres et de logiques différents. Les fils narratifs principaux sont au nombre de trois : il y a d'abord le présent du narrateur comateux Roy Strang, dans le service de soins de longue durée d'un hôpital anonyme, puis le récit, sur le mode réaliste, de son enfance et de sa vie adulte jusqu'à la tentative de suicide responsable de son état et, enfin, l'univers fantastique dans lequel il se projette pour fuir l'abêtissement de son quotidien et la violence de son passé. Cette troisième réalité abandonne complètement le registre réaliste du récit de vie : elle tente plutôt d'en sublimer les tristes conditions, celles d'un Édimbourg suburbain et paupérisé. Elle prend les traits d'une savane africaine, décor au sein duquel Roy s'arroge un pouvoir dont il est en vérité totalement dénué en s'inventant aventurier colonial et chasseur émérite de bêtes étrangement familières. Car malheureusement pour le rêveur, le rêve rejoint bientôt le monde réel, c'est-à-dire que les événements qui s'y déroulent sont fortement influencés par l'histoire individuelle du protagoniste et l'histoire nationale dont il participe. Cependant, Roy étant amnésique, ces échanges et la véracité des faits auxquels ils renvoient sont instables. Le lecteur ne réalise ainsi qu'à la toute fin que le viol collectif dont la responsabilité est attribuée tout au long du roman à un autre a en fait été commis à l'instigation du narrateur lui-même. Cette culpabilité est appuyée par la coïncidence finale entre Roy et le marabout éponyme, prédateur féroce et symbole du mal dont il s'était donné pour mission de purger le monde imaginaire. 
Un tel résumé est difficile à réaliser, rejoignant ainsi l'un des constats posés par Dominique Rabaté à propos de ce qu'il nomme la «littérature de l'épuisement»: "Contrairement à bien d'autres romans plus "classiques" où la fiction se déploie dans un espace imaginaire autre que régissent les règles d'un faire-semblant, ces livres ne laissent pas isoler des épisodes fictifs précis: ils ne se prêtent pas aux mêmes formes de résumé. » (p. 7) Le problème, dans Marabou Stork Nightmares, est déplacé: les épisodes précis sont nombreux, mais ils se répètent à plusieurs reprises et sous diverses formes dans des espaces imaginaires pluriels, régis chacun par des règles changeantes. Ce foisonnement et, surtout, l'impossibilité, pour le lecteur, d'y discerner le faux du vrai en raison des défauts de mémoire du narrateur sont les bases de l'épuisement de la partie réaliste du récit de Welsh. En contrepartie, sa dimension fantaisiste propose une mise en récit de l'histoire écossaise et britannique, par le biais d'éléments symboliques à décrypter. Cela conduit à une représentation sociale traversant les niveaux narratifs du roman, au moyen de la pérennité de la figure du marabout et des images du viol. Le commentaire ainsi posé sur le réel contemporain et plus ancien est complété par une entreprise d'exégèse littéraire. La dichotomie entre les registres réaliste et fantastique, si elle reproduit les traits de la littérature mineure établis par Deleuze et Guattari, comme on le notera au fil de l'analyse, offre plus précisément une mise en abyme de la littérature écossaise dans son ensemble et, surtout, des contradictions censées la caractériser.

\section{Le réalisme corrompu}

Si contradiction il y a dans Marabou Stork Nightmares, c'est avant tout dans l'énoncé de base des faits en constituant la 
trame événementielle, qui perturbe complètement le récit de vie aux apparences réalistes. Roy Strang est amnésique, comme il l'avoue dès la deuxième page - «my memory is practically non-existent ${ }^{1}$ (p. 4), dit-il —, puis le répète à plusieurs reprises. De plus, il fuit résolument la vérité, vérité du crime qu'il a commis, mais aussi de son quotidien guère réjouissant. Cela en fait un narrateur particulièrement peu fiable, dont la moindre des affirmations est à mettre en question : l'exemple de la responsabilité du viol donné plus haut en témoigne. Mais l'incertitude ne touche pas seulement des épisodes tragiques comme celui-là. Ainsi, il est dit à la dernière page que Roy " never really had much in the way of ears, it was always [his] nose, Captain Beaky, they used to call [him] at the school ${ }^{2}$ (p. 264). Pourtant, jusque-là, ce sont justement ses oreilles protubérantes, à l'origine du surnom «Dumbo Strang», qui étaient mentionnées avec redondance parce qu'elles l'embarrassaient. Les moqueries de camarades de classe à ce sujet auraient d'ailleurs été à l'origine de ses premières exactions et de sa première agression à caractère sexuel sur une jeune fille. Le renversement est donc significatif, d'autant que son «bec» aiguisé est une caractéristique majeure du marabout : est resserré le lien physique entre Roy et l'oiseau prédateur, préalablement établi par leur claudication et leur laideur communes. L'image agonisante de lui-même dressée par Roy reprend d'ailleurs exactement les termes de sa vision initiale de son ennemi et alter ego : les deux tournent le dos au soleil dans un contre-jour dramatique et le grand manteau

\footnotetext{
1 «Ma mémoire est presque inexistante.» Toutes les traductions sont effectuées par moi.

${ }^{2}$ Roy «n'a jamais eu de grandes oreilles, c'était toujours [son] nez, à l'école on [l']appelait Capitaine Beaky. »
} 
(«large overcoat», p. 264) de l'homme évoque les ailes déployées («spread [...] wings», p. 14) du volatile.

Pour peu qu'on se donne la peine d'en examiner les ramifications, le détail physique anodin prend des proportions insoupçonnées en regard de l'intrigue globale. Celle-ci étant narrée selon une focalisation interne exclusive - puisque prise en charge par un personnage physiologiquement coupé du monde et refusant la confrontation de son discours à tout autre - , le lecteur n'a aucun moyen d'en vérifier la véracité. Mieke Bal explique que la focalisation, en tant que relation entre la "vision», l'agent qui voit et ce qu'il voit, influence l'histoire et ce que le lecteur en perçoit (p. 104). Dans le cas présent, la simple possibilité d'une adéquation entre objet vu et vision est remise en question, l'agent se permettant de la pervertir sans en avertir le lecteur. Semblable disjonction est toujours présente en prose romanesque, mais ici, elle devient la règle, de manière à pousser à ses limites les conditions de cette prose et en proposer, en quelque sorte, l'épuisement. Le phénomène est accentué par l'artificialité consciente du récit, qui combine réalisme social et jeux autoréflexifs qui en dévoilent les soubassements. Le premier est un peu la marque de commerce de Welsh. Auteur à succès taxé à l'occasion de sensationnalisme et d'une certaine dose de voyeurisme, il bâtit sa popularité sur une approche "sans concession» de réalités sociales difficiles, notamment celle des périphéries défavorisées d'Édimbourg. L'auteur cherche à ramener au grand jour ce que l'image officielle et avant tout culturelle de la capitale écossaise tend à occulter, soit un chômage endémique et des problèmes de drogue, de violence urbaine et d'éclatement familial. Dans Marabou Stork Nightmares, l'exigence de vérité à tout prix est à la base de recoupements biographiques entre l'enfance de Roy 
et de ses amis et celle de Welsh, du moins telle qu'il la raconte. De nombreux passages du roman répondent directement aux dires de l'auteur dans différentes entrevues; de plus, ses remerciements, en avant-propos, sont adressés à des gens qui lui ont fourni de l'information ou encore de l'information spécialisée. Cette insistance sur le caractère documentaire et documenté du roman - dont il ne faut pas oublier qu'il comporte un côté complètement fantaisiste et qu'il se déroule entièrement dans la tête d'un narrateur comateux et fabulateur-,a poussé certains critiques comme Marina MacKay à le qualifier d'«anthropologique» (p. 269-81). Le terme n'est pas négligeable : en sus de l'observation de données singulières, il renvoie à leur analyse et donc à la généralisation.

La part d'observation est visible dans l'attention portée au langage, qui reproduit phonétiquement les accents et intonations des personnages. La plupart d'entre eux parlant le scots, dialecte proche de l'anglais mais qui s'en distingue par quelques traits syntaxiques et variations de vocabulaire, le résultat est de prime abord déstabilisant pour les non-initiés. «Yes», «I don't» et "I know »3 deviennent «Aye», «Ah dinnae » et «Ah ken»; les mais («but») se placent en fin de phrase plutôt qu'au début, et ainsi de suite. Ces divergences par rapport à l'anglais standard peuvent être associées aux «étranges usages mineurs» qui, selon Deleuze et Guattari, visent l'appropriation d'une langue qu'on ne sent pas sienne (rappelons en effet que l'anglais est imposé à l'Écosse, initialement gaélique). Dans leur théorisation de la littérature mineure, les deux auteurs rappellent qu'elle " n'est pas celle d'une langue mineure, plutôt celle qu'une minorité fait dans une

\footnotetext{
3 « Oui », « Je ne » et « Je sais ».
} 
langue majeure. Mais le premier caractère est de toute façon que la langue y est affectée d'un fort coefficient de déterritorialisation » (p. 29). Celle-ci est certainement présente chez Welsh, dont on a pu dire que les déviations phonétiques « infectaient» l'imagination du lecteur en l'obligeant à prononcer mentalement les mots pour comprendre (voir Christopher, 1995, p. 18). Cette déterritorialisation linguistique, cependant, n'est pas toujours motivée : certains termes sont orthographiés d'une manière qui ne change rien à leur prononciation : entre "there » (là) et " thair », il ne semble pas y avoir grande différence. L'observation «anthropologique» mène donc, sur le plan de la langue, à la généralisation d'une étrangeté idiomatique exagérée. Le constat vaut aussi pour les protagonistes : leurs traits sont grossis à l'extrême, en faisant des figures, des caractères davantage que des personnages.

La famille de Roy en particulier, qu'Alan Freeman qualifie de collection de pathologies émotionnelles gravitant autour d'un nom partagé (p.139), ressemble davantage aux marionnettes d'un guignol macabre qu'à un ensemble humain. Cette impression caricaturale est évidente dès leur description physique, commune: "'The Strang look' was essentially a concave face starting at a prominent, pointed forehead, swinging at a sharp angle towards large, dulled eyes and a small, squashed nose, down into thin, twisted lips and springing outwards to the tip of a large, jutting chin. $»^{4}$ (p. 20) L'anatomie grotesque est complétée par une absence totale d'intériorité, constituant les membres de la famille en porte-étendards

\footnotetext{
4 " "Le type Strang" consistait essentiellement en un faciès concave qui commençait avec un front proéminent et pointu, rejoignait à angles aigus de grands yeux vides et un petit nez écrasé, descendait vers de fines lèvres tordues puis s'évasait au gré des extrémités d'un menton trop large. »
} 
indifférenciés d'une série de problèmes sociaux caractéristiques de leur milieu défavorisé. Le père devient risible, tant il est violent et obtus. Quant à la mère, sa haine paranoïaque des Japonais souligne à gros traits les séquelles de la Seconde Guerre mondiale sur l'imaginaire britannique, duquel le roman s'attache à démontrer les liens ambigus avec l'Écosse. MacKay explique en effet que tous les préjudices raciaux qui y sont exposés - comme celui-ci - sont à relier à des traumatismes vécus par la Grande-Bretagne (p. 276-7). L'entreprise documentaire est donc à la fois menée à son aboutissement et corrompue, en ce que les personnages n'ont d'existence qu'en fonction de phénomènes extérieurs. Cette tendance à l'extrême généralisation vaut pour le personnel romanesque comme pour les épisodes, qui ne sont pas tant à considérer pour eux-mêmes qu'en ce qu'ils représentent. Ainsi, lorsque Roy décrit les minuscules prisons où il se plaît, enfant, à enfermer des abeilles: "We had a concentration camp, a tiny Scottish housing scheme, for bees. $»^{5}$ (p. 22) L'association entre HLM et milieux concentrationnaires est réitérée plus loin, lorsque les mêmes "schemes » sont à leur tour comparés à des camps autosuffisants (« self-contained camps», p. 80). Le cruel passe-temps enfantin est donc assimilable à une forme de masochisme social, Roy s'attaquant, symboliquement, à son propre milieu. Cela rejoint une réalisation future : alors qu'il serait sensé de s'attaquer aux exploiteurs, "we screw each other's hooses when there's fuck all in them, we terrorise oor ain people $»^{6}$ (p. 201).

\footnotetext{
5 « Nous avions un camp de concentration, un minuscule HLM écossais, pour abeilles.»

6 « Nous vandalisons les maisons de nos voisins qui contiennent que dalle, nous terrorisons notre propre milieu. »
} 
Plus largement, la métaphore des abeilles en vient à désigner l'Écosse entière, au travers d'un riche réseau intertextuel. Leur petit camp de concentration renvoie explicitement à un auteur contemporain de Welsh, son compatriote Iain Banks, dont Wasp Factory développe aussi une société en microcosme. Le jeune protagoniste y vit sur une île isolée, où il peuple son existence de différents rituels, parmi lesquels l'usine à guêpes éponyme. Les deux romans mettent également en scène des pères dégénérés et des molosses cruels, doubles déchus des chiens écossais fidèles fièrement représentés par Lassie et Greyfriars Bobby. La reprise en écho de ces éléments participe dans les deux cas d'une peinture sociale sombre, où les valeurs de filiation et de loyauté sont vidées de sens. De la même manière, le parcours de Roy peut être interprété comme un déboulonnage en règle du mythe du Hard Man écossais. La figure trouve son expression la plus achevée dans Docherty, de William Mcllvanney (1975), qui suit un mineur dans sa lutte pour la survie et la dignité de sa famille. Comme lui, Roy cherche à s'affirmer par des moyens essentiellement physiques et une virilité appuyée; cependant, il va trop loin et ne parvient qu'à détruire son entourage et luimême. Le coma qui en résulte rejoint un autre thème largement répandu dans la littérature écossaise contemporaine : l'immobilisation forcée du protagoniste (on peut penser ici à 1982 Janine d'Alasdair Gray ou à The Bridge d'Iain Banks, dans lequel un personnage accidenté et inconscient se projette d'ailleurs également dans un univers parallèle.

Ce jeu intertextuel, s'il enrichit le récit, concourt à en dévoiler les artifices et ainsi à miner l'effet de réalisme social précédemment décrit : le texte se prend pour objet, annulant une adhésion possible du lecteur à la réalité de son monde 
diégétique. Il se positionne également vis-à-vis de la tradition littéraire nationale, en récupérant certains éléments de manière à réfléchir sur cette tradition et sur la réalité écossaise contemporaine. Welsh n'est pas le seul à se livrer à l'exercice : Cairns Craig affirme, par exemple, que la fixité des personnages est une représentation récurrente de la paralysie et de l'immobilisme de l'Écosse moderne (p. 132). Son coma assure une continuité entre Roy et les héros d'Iain Banks et d'Alasdair Gray, ancrant le roman dans une énonciation de portée nationale. Il s'agit là d'une autre condition de la littérature mineure selon la définition de Deleuze et Guattari, qui arguent que «tout $[y]$ prend une valeur collective », dans un contexte où l'énonciation individuée d'un « maître » n'est pas discernable de la voix commune (p. 31). Même seul, l'écrivain participe d'une action qui le dépasse, ainsi que le montre, chez Welsh, le dialogue actif avec la littérature écossaise. En plus d'assurer une continuité entre l'auteur et d'autres écrivains, cet échange éclaire l'œuvre d'un jour nouveau et précise son discours, comme le montrent les exemples du coma ou du Hard Man. Ceux-ci ouvrent l'incident personnel sur l'idéologique et le politique, ce qui constitue la dernière caractéristique de la littérature mineure : "Son espace exigu fait que chaque affaire individuelle est immédiatement branchée sur la politique. L'affaire individuelle devient donc d'autant plus nécessaire, indispensable, grossie au microscope qu'une tout autre histoire s'agite en elle. » (Deleuze et Guattari, 1975, p. 30) C'est ce qui se produit dans Marabou Stork Nightmares, où la trame fantaisiste résume à la fois une partie de l'histoire écossaise et la vie de Roy, ainsi qu'il la raconte dans le récit réaliste parallèle. 


\section{Le véridique dans l'imaginaire}

L'épuisement de celui-ci est donc corollaire de la mise en récit du réel déployée dans le monde imaginaire. Cette tendance est notamment à l'œuvre dans la construction, au moyen d'homologues réels, des protagonistes qui peuplent les fantasmes comateux de Roy. Il s'agit, en fait, d'un amalgame de figures du réel extra- ou intradiégétique. La concaténation entre elles se produit malgré le narrateur, celui-ci cherchant désespérément à fuir, dans le monde imaginaire, le présent de l'hôpital et le passé de ses souvenirs. Est exemplaire de ce double mouvement le fait que Roy se réfère à Sandy, son compagnon de chasse, à la fois comme à une métaphore (une chose qui en représente une autre) et un ami (p.6). Le narrateur s'avoue ainsi créateur du personnage, dans une mise en abyme ludique du processus d'écriture qui en affirme encore l'épuisement. Le nom de Sandy Jamieson constitue un remaniement de celui du (vrai) footballeur Jimmy Sandison, dont le visage à la télévision est la dernière image vue par Roy avant de sombrer dans l'inconscience, lors de son suicide manqué. La référence importe dans l'univers mental une allusion au hooliganisme de Roy, propension à la criminalité qui s'incarne dans le personnage, lui aussi imaginé, de Lochart Dawson. Robert Morace explique que celui-ci, propriétaire tyrannique d'un parc naturel, est un agrégat de l'avocat Donaldson, de l'oncle Gordon et de Wallace Mercer, le véritable président et actionnaire principal du club de football des Hearts of Midlothian (p. 110). Les noms des deux premiers résonnent par allitération dans celui de Dawson, qui partage leur caractère véreux, Gordon, comme lui, étant un Afrikaner. Ils sont connus personnellement de Roy: le premier le défend efficacement en cour quand il est accusé de viol, et le second 
l'agresse de la même manière lorsqu'il est jeune garçon. Les deux se retrouvent donc, en quelque sorte, aux antipodes de l'évolution sexuelle de Roy; l'un l'ouvre, l'autre la ferme et répare les dégâts. Le roman prend fermement position quant au lien de causalité entre les deux : un crime en appelle un second, au cours d'un cycle inexorable de violence. Ce discours, ailleurs explicite, ne se trouve nulle part validé avec autant de cohérence que dans la cohabitation, à l'intérieur d'un même protagoniste, des instances du viol subi et du viol commis. Leur relation est scellée sans retour, ainsi que celle de cette forme d'agression à l'exploitation capitaliste.

Ce dernier élément de l'équation est apporté par la présence du propriétaire des Hearts qui, en 1990, tenta de manière déloyale de prendre le contrôle du club rival des Hibernians, agissements représentés dans le récit par les manigances de Lochart Dawson en vue d'un agrandissement territorial et par la relation d'identité qu'il pose entre sport et quête de profits. Roy se tient du côté des victimes de la manœuvre, surnommés les «Hibs », contre l'équipe de Mercer, les « Jambos », desquels le nom du parc de Dawson, Jambola, est inspiré, position de faiblesse qui reflète son infériorité économique réelle. La division va plus loin, cependant : les Hibs étant catholiques d'Édimbourg qui affrontent périodiquement les Jambos, protestants de la capitale, ou les clubs catholiques et protestants de Glasgow, c'est la scission religieuse et culturelle écossaise qui est représentée. Le détail serait anecdotique si ce n'était le numéro de la retraite de Dawson dans les montagnes : 1690, soit l'année où Guillaume d'Orange défit le Catholique James Stuart à la bataille de la Boyne, une victoire célébrée annuellement par les Unionistes protestants (partisans des Jambos). En somme, c'est la position de l'Écosse vis-à-vis de la 
Grande-Bretagne qui est métaphoriquement esquissée ici, comme elle l'est d'ailleurs à de multiples reprises dans l'espace fictif de la savane.

Le cas de Lochart Dawson est exemplaire de la fluidité constitutive des figures imaginées : communiquent en son sein des personnages appartenant au réel extradiégétique (Wallace Mercer et les acteurs d'une histoire écossaise pré-unioniste) et des protagonistes intradiégétiques (l'avocat Donaldson et Gordon). Le phénomène est indicateur d'une circulation sémantique à grande échelle entre les différentes réalités du roman, circulation qui se concrétise dans l'univers mental de Roy. Celui-ci englobe les autres couches narratives de façon symbolique et, au-delà, le récit national. Cette mise en fiction de l'histoire associe les événements vécus individuellement par les personnages à une trame beaucoup plus vaste, afin d'expliquer — ou du moins mieux comprendre — les violences auxquelles ils se livrent. Ainsi, les fantasmes de Roy sont parsemés de références à l'agression qu'il a commise: un contenu violent et érotique en envahit progressivement la narration, et ce, en dépit des efforts du narrateur de le proscrire en changeant brusquement de sujet ou en "s'enfonçant»comme cela est signifié sur la page par des mouvements descendants de la typographie - plus profondément dans ses rêveries. La concomitance des discours investit l'histoire sociale d'une charge de brutalité sexuelle, lien scellé par la récurrence de mêmes images dans des situations différentes. La dévoration de flamands par des marabouts revient notamment à plusieurs reprises: enfant, Roy est témoin du spectacle alors qu'il découvre la domination coloniale lors d'un voyage dans l'Afrique du Sud ségrégationniste, et il en a à nouveau la vision dans son monde imaginaire. Les descriptions de ces 
événements font écho à celle de la jeune fille qu'il agresse, immédiatement après le viol: il est toujours question de « beauté » volée et détruite par des prédateurs repoussants, au cours d'une attaque particulièrement cruelle.

Par le biais de la figure polysémique du marabout, le viol est placé au centre d'un réseau plus large de violence, c'est-àdire que l'agression sexuelle et l'humiliation qui en résulte règlent aussi les échanges sociaux décrits dans le roman. Les rapports de pouvoir qui régissent les liens du violeur à la victime recoupent ceux qui divisent l'ensemble de la communauté en actifs et passifs, entre agresseurs et agressés. Le viol devient ainsi métaphore sociale, comme le confirme l'adhésion à son endroit du discours juridique lors de l'épisode judiciaire. Face aux jurés, Kirsty, la victime, se retrouve en position d'accusée : c'est son procès à elle qui est fait plus que celui de ses violeurs, puisqu'on la questionne sans merci sur sa sexualité et sur ses mœurs. Le jugement ne lui est pas favorable et, par la suite, elle s'y réfère plusieurs fois comme à un "second viol», reprenant ainsi sur le mode tragique l'expression malheureuse de l'avocat des agresseurs, qui, à la veille de leur passage en cour, promet à ses clients : «we'll give her a damn good shafting $»^{7}$ (p. 207). L'usage récurrent de ce vocabulaire à forte consonance sexuelle appuie la symétrie entre viol et société; dans ce contexte, il est significatif que Roy se réfère initialement à son milieu d'origine comme à «that fucked-up place which made me the fucked-up mess I was ${ }^{8}$ (p. 16-17). Le lien causal établi entre les deux jurons peut être

\footnotetext{
7 « Nous allons la baiser en beauté ».

8 « l'endroit tordu qui avait fait de moi l'inadapté tordu que j'étais devenu ». Je souligne; il est à noter que le mot «fuck» est porteur, en anglais, d'une polysémie et d'une vulgarité intraduisibles.
} 
appliqué aux viols eux-mêmes : ayant été violé, Roy devient violeur, ainsi que le confirme l'existence, dans le monde imaginaire, de Lochart Dawson. Et c'est pourquoi il n'y a, au terme de la poursuite du marabout, que lui-même à la pointe du fusil...

\section{La littérature dans tous ses états}

Le thème de la chasse, qui traverse les rêveries comateuses du personnage, est assimilé au viol et à la domination raciale, mais aussi à la violence hooligan : un chapitre entier, « The Flamingo Massacres », utilise une narration alternée pour mettre en parallèle le massacre des flamants et celui, par le gang dont Roy fait partie, d'une bande rivale. Le lien avec le viol est lui aussi posé par l'attribution de formes féminines aux cadavres des animaux. La circulation de la même image fait du marabout un symbole polysémique qui représente toutes les formes d'agression du roman et qui en traverse ainsi toutes les couches narratives, réelles ou non. Le monde animal en vient à désigner celui des hommes, à la fois dans leurs comportements personnels et en communauté. Cette métaphorisation fantaisiste de la réalité cohabite, au sein du roman, avec la visée réaliste de la peinture d'un milieu social particulier et de ses conditions propres. Le contraste formel qui en résulte est très marqué. Il serait aussi, explique Alan Riach, fondateur de l'œuvre tout entière :

The fiction of Irvine Welsh offsets the mimetic representation of reality by combining two apparently contradictory modes: an immediate vernacular voice, ostensibly an authentic representation of location-specific speech, and a narrative 
where unpalatable basic realities of economic and bodily imperatives are paramount. ${ }^{9}$ (p. 35-6)

Or, dans The Scottish Novel: A Critical Survey, Francis Russell Hart place semblable contradiction générique au cœur du roman écossais depuis ses origines; dès Smollet en effet, au XVIII ${ }^{\text {e }}$ siècle, se donnerait à lire une indécision entre les modes du «Gothic fantasy» et de l'histoire sociale (p. 398). Cette dichotomie essentielle serait due, selon Hart, à la conception complexe et duelle du «caractère national » de l'Écosse - à relier à sa relation difficultueuse avec l'Angleterre - et à la divergence des traditions écrite et orale qui fondent sa littérature. La «bipolarité» évoquée par Hart est caractéristique de l'histoire de la littérature de l'Écosse par ellemême, comme l'expliquent Gerard Carruthers, David Goldie et Alastair Renfrew. En effet, celle-ci est représentée par ses propres exégètes comme « a gargoyle grinning at the elbow of a kneeling saint $»^{10}$ (p. 11).

L'œuvre de Welsh, en plus de réaliser les conditions de la littérature mineure en général, reprend donc à outrance les caractéristiques distinctives d'une littérature mineure singulière : la sienne. Les citations intertextuelles se doublent d'une intertextualité formelle, en diversifiant et en approfondissant les effets. Ainsi non seulement Marabou Stork Nightmares représente-t-il par la fiction la position ambiguë de l'Écosse dans le Royaume-Uni, mais il le fait aussi dans sa

\footnotetext{
9 « L'œuvre de fiction d'Irvine Welsh décale la représentation mimétique de la réalité en combinant deux modes apparemment contradictoires : une parole vernaculaire immédiate, représentation ostensible d'un discours local authentique, et une narration où les retombées désagréables de divers impératifs physiologiques et économiques sont prépondérantes. »

10 « une gargouille souriant par-dessus l'épaule d'un saint agenouillé ».
} 
structure narrative, mettant sa propre histoire culturelle en abyme. Les scissions, aujourd'hui critiquées, qui constitueraient la base de celle-ci sont toutefois déplacées: le «réalisme social » s'épuise dans la généralisation, tout en étant miné par l'autoréflexivité d'un jeu intertextuel qui en dévoile les artifices; quant à l'aspect fantastique, il ne l'est pas autant qu'il le semble au premier abord, étant donné qu'il procède par la bande à une mise en récit de l'histoire au moyen d'éléments symboliques. C'est peut-être dans ce jeu formel que l'entreprise de dévoilement de Welsh, qu'on a vu présente dans sa narration réaliste d'un milieu difficile, donne les meilleurs résultats. En convoquant le réel dans un espace purement imaginaire et en le soumettant à des règles qui n'ont rien de réaliste, le roman l'interprète à sa manière et fait table rase des oppositions de genres consacrées. C'est sa manière à lui d'affirmer sa position «mineure», soit, pour citer une dernière fois Deleuze et Guattari, de réaliser «les conditions révolutionnaires de toute littérature au sein de celle qu'on appelle grande (ou établie) » (p. 33).

\section{Bibliographie}

BAL, Mieke. (1985 [1980]), Narratology: Introduction to the Theory of Narrative, trad. Christine van Boheemen, Toronto, University of Toronto Press.

CARruthers, Gerard, David Goldie et Alastair REnFREW. (2004), Beyond Scotland: New Contexts for Twentieth-Century Scottish Literature, Amsterdam, New York, Rodopi. 
Christopher, James. (1995), «Blood on the tracks », Time Out, 12-19 avril, p. 18-20.

CrAIG, Cairns. (1999), The Modern Scottish Novel: Narrative and the National Imagination, Edinburgh, Edinburgh University Press.

Deleuze, Gilles et Félix Guattari. (1975), Kafka. Pour une littérature mineure, Paris, Minuit, coll. « Critique ».

FREEMAN, Alan. (1996), "Ourselves as Others: Marabou Stork Nightmares», Edinburgh Review, Edinburgh University Press, no 95, p. 135-141.

HART, Francis Russell. (1978), The Scottish Novel: A Critical Survey, Londres, John Murray.

MACKAY, Marina. (2003), «Marabou Stork Nightmares: Irvine Welsh's Anthropological Vision», National Identities, vol. 5, no 3, p. 269-281.

MoraCE, Robert A. (2007), Irvine Welsh, Houdmills, Basingstoke, Palgrave MacMillan, coll. « New British fiction».

RABATÉ, Dominique. (1991), Vers une littérature de l'épuisement, Paris, José Corti, coll. « Les Essais ».

RiACH, Alan. (2005), "The Unnatural Scene: The Fiction of Irvine Welsh », dans J. ACHESON et Sarah C. E. Ross (dir.), The Contemporary British Novel Since 1980, Edinburgh, Edinburgh University Press, p. 35-47.

WELSH, Irvine. (1996 [1995]) Marabou Stork Nightmares, Londres, Vintage. 


\title{
Résumé
}

La littérature prend acte de la pauvreté ressentie du réel contemporain en optant pour l'épuisement, tandis que les discours déployés dans l'espace public, pour pallier cette même pauvreté, deviennent le lieu d'une narrativisation croissante. Ces phénomènes, complémentaires, sont toutefois difficiles à appréhender simultanément: ils relèvent de domaines différents. $\mathrm{Au}$ moyen de l'exemple de Marabou Stork Nightmares d'Irvine Welsh (1995), je montrerai que le roman peut, cependant, rendre compte de ce double jeu. Le réalisme affiché y est corrompu par l'amnésie du narrateur, tandis que la dimension fantaisiste du roman propose une mise en récit de l'histoire écossaise et britannique qui conduit à une représentation sociale et littéraire en traversant les trames.

\begin{abstract}
Literature expresses the poverty of our contemporary reality by its own exhaustion, whereas discourses displayed in the public space, to mitigate the same poverty, are increasingly narrative. These phenomena, though complementary, are difficult to comprehend simultaneously: they pertain to different domains. Using the example of Irvine Welsh's Marabou Stork Nightmares (1995), I shall show that the novel can, however, take this double game into account. The realism displayed is corrupted by the narrator's amnesia, whereas the novel's fantastic dimension proposes a symbolic display of Scottish and British history, leading to a social and literary representation crossing its levels.
\end{abstract}

